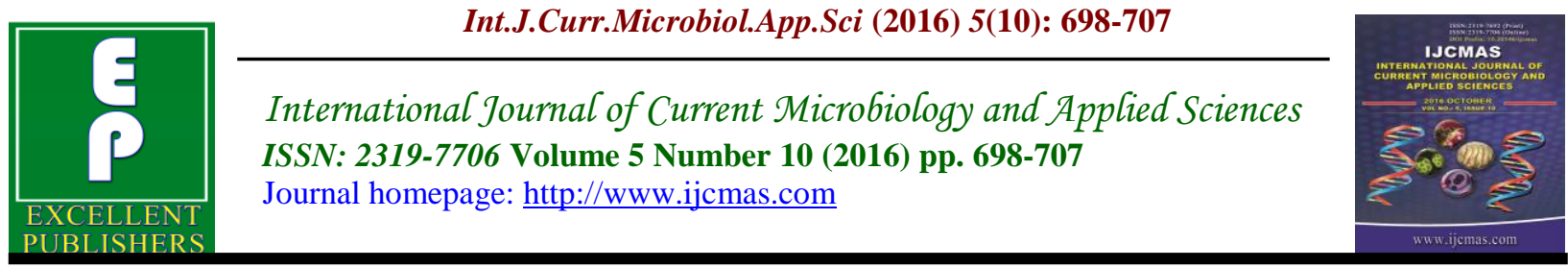

Original Research Article

http://dx.doi.org/10.20546/ijcmas.2016.510.076

\title{
Isolation and Identification of Some Dark Septate Endophytic Fungi (DSE) from the Roots of the Ethno-Medicinal Plant Plumbago zeylanica L.
}

\author{
Avishek Sarkar ${ }^{1}$ and Sikha Dutta* \\ CAS Department of Botany, The University of Burdwan, Burdwan-713104, West Bengal, India \\ *Corresponding author
}

\section{A B S T R A C T}

Keywords

Dark septate endophytic fungi; Perenniporia sp.; Aspergillus niger; Plumbago zeylanica $\mathrm{L}$.

\begin{tabular}{l}
\hline Article Info \\
\hline Accepted: \\
20 September 2016 \\
Available Online: \\
10 October 2016
\end{tabular}

In present study an attempt has been made to isolate and identify the dark septate endophytic fungi (DSE) and their colonization in the roots of an important ethno-medicinal plant, Plumbago zeylanica L. The plant exhibited significant colonization by DSE. Two different strains of dark septate endophytic fungi were isolated from the roots of the plant. The pure cultures were established (PUB and PUD). DNA was isolated from the Pure culture - PUB and PUD and 28s rDNA sequencing of $\mathrm{D}_{1}, \mathrm{D}_{2}$ region of the larger sub unit was performed. Sequence analysis exhibited sequence similarity with Perenniporia sp. CBS 130020 (Gen Bank Accession Number: JX292098.1) and Aspergillus niger strain RA402 (GenBank Accession Number: GQ169753.1) respectively based on nucleotide homology and phylogenetic analysis.

\section{Introduction}

Vascular plants host a great variety of fungi. In addition to being susceptible to soil-borne pathogens, plant roots are also colonized by non-pathogenic or mutualistic fungi like arbuscular mycorrhizae (AM), ectomycorrhizae (EM) and dark septate endophytic fungi (DSE). A vast majority of terrestrial plant species form mycorrhizal associations (Harley and Smith, 1983, Smith and Read, 1997). It is now evident that the mycorrhizal fungi have many significant functions in ecosystem like they absorb nonmobile nutrients from the soil and translocate them to host plants, sequester potentially harmful heavy metal ions, facilitate interplant transfer of nutrients, and beneficially modify plant water relations (Smith and Read, 1997).

In contrast to the surfeit of knowledge about the EM and AM fungi, very little is known about the DSE. The DSE are broadly classified as conidial or sterile septate fungal endophytes that form melanized structures such as inter- and intracellular hyphae and microsclerotia within the plant roots and that have known or likely affinities with ascomycetes (Jumpponen and Trappe, 1998). 
DSE are found worldwide and coexist often with different mycorrhizal fungi. They have been reported from many plant species including plants that have been considered non-mycorrhizal (Jumpponen and Trappe, 1998).

DSE fungi under some environmental or experimental conditions, can enhance host growth and nutrient uptake, hence functioning in a manner typical of mycorrhizal associations. Enhanced growth and improved nutritional status indicate better performance resulting from DSE colonization. It has been suggested that the microsclerotia may serve as dispersal structures (Currah and Tsuneda, 1993), although the actual purpose appears to be unknown. Perhaps they serve a storage function for the fungi as do the vesicles of AM fungi.

There are three different mechanisms through which DSE fungi may inhibit pathogens, or minimize their impact on plant growth and performance. Firstly, mycorrhizal fungi and rhizosphereinhabiting pathogens may compete for the same recourse of plant photosynthates or for sites of colonization. Secondly, compounds inhibitory to pathogens may be produced. Finally, the DSE colonization may have prophylactic value by inducing plant defense responses to subsequent pathogen infection (Mandyam and Jumpponen, 2005). Some strains of DSE may be involved in host plant nutrient acquisition and it has been proposed that this may be a mutualistic, mycorrhizalike relationship which do not appear to be host specific (Jumpponen and Trappe, 1998),

Research works on DSE colonization in various groups of plants is still limited. Hence, in present investigation, our endeavor has impelled us to isolate and identify the DSE form the roots of
Plumbago zeylanica L. an ethno medicinally important herb having strong female abortificant property.

\section{Materials and Methods}

Secondary and tertiary roots were collected from the plant Plumbago zeylanica L., Freshly collected roots were rinsed in distilled water for several times to remove all the soil debris. Surface sterilization of the roots was done with ethyl alcohol, $0.01 \%$ $\mathrm{NaOCl}$ and antibiotic water successively to disinfect the roots and to kill the bacteria and fungal spores remaining outside.

Roots were cut opened in $\mathrm{Y}$ shape with the help of a sterilized blade, placed in sterilized PDA media supplemented with antibiotic to prevent any Bacterial growth.

After observing fungal growth, pure culture was established by serial dilution method.

DNA was isolated from the Pure culture PUB and PUD. Quality was evaluated on $1.0 \%$ Agarose Gel, a single band of highmolecular weight DNA was observed.

Fragment of D2 region of $28 S$ rDNA was amplified by PCR from the above isolated genomic DNA. A single discrete PCR amplicon band of 700bp was observed when resolved on Agarose Gel (Figure 1a,b respectively)

The PCR amplicon was purified and further processed for the sequencing.

Forward and Reverse DNA sequencing reaction of PCR amplicon was carried out with DF and DR indicator and (DF:ACCCGCTGAACTTAAGC, DR: GGT CCG TGT TTC AAG ACG G) primers using BDT v3.1 Cycle sequencing kit on ABI 3730xl Genetic Analyzer. 
Consensus sequence of 641 bp of D2 region of LSU gene was generated from forward and reverse sequence data using aligner software.

The D2 region of $28 S$ rDNA sequence was used to carry out BLAST with the nrdatabase of NCBI genbank database. Based on maximum identity score, fifteen sequences were selected and the phylogenetic tree was constructed using MEGA 5.

The evolutionary history was inferred using the Neighbor-Joining method [Felsenstein, 1985]. The bootstrap consensus tree inferred from 1000 replicates [Harley et al., 1983] is taken to represent the evolutionary history of the taxa analyzed [Harley et al., 1983].

\section{Results and Discussion}

The culture, labeled as PUB exhibited sequence similarity to Perenniporia sp. CBS 130020 GenBank Accession Number: JX292098.1) and the culture, PUD to Aspergillus niger strain RA402 (GenBank Accession Number: GQ169753.1) based on nucleotide homology and phylogenetic analysis.

Information about other close homologs for the microbes can be found from the Alignment View table and from the phylogenetic tree.

Fig.1 1.0\% Agarose gel showing 700bp amplicon (D2 region) of 28S rDNA

Quality Check on 1.0\% Agarose gel

a

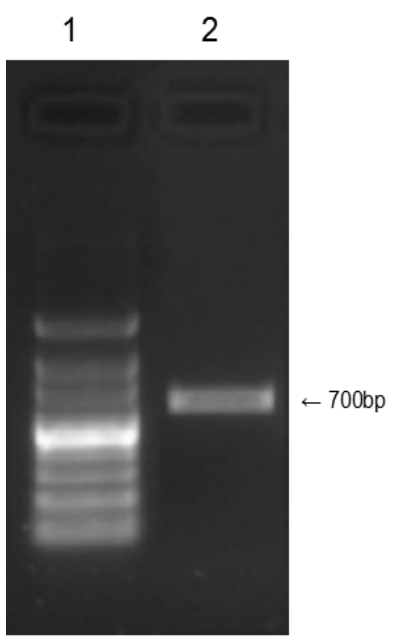

b

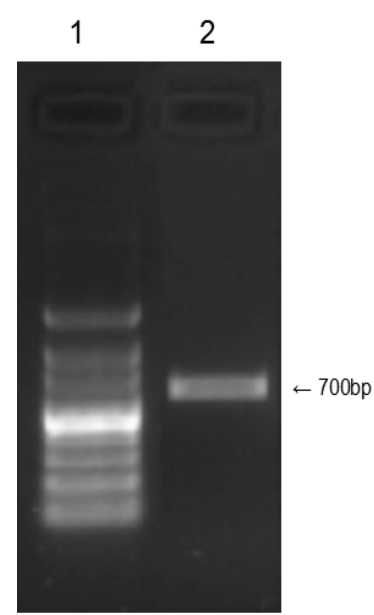

Lane 1: $1 \mathrm{~Kb}$ DNA Ladder

Lane 2: 700bp amplicon (D2 region) of $28 S$ rDNA 
Sequence data of the sample PUB

1. PUB-2B_DF_S014381_A05_047.ab1: Data obtained with Forward primer 2. PUB-2B_DR_S014381_B05_045.ab1: Data obtained with Reverse primer PUB-2B_DF_S014381_A05_047 (565 bp)

GCTCAAATTTAAAATCTGGCGGTCTTTGGCCGTCCGAGTTGTAGTCTGGAGAAGTGCTTTCCGCGCTGG ACCGTGTATAAGTCTCTTGGAACAGAGCGTCGTAGAGGGTGAGAATCCCGTCTTTGACACGGACTACC AGTGCTTTGTGATGCGCTCTCAAAGAGTCGAGTTGTTTGGGAATGCAGCTCAAAATGGGTGGTAAATT CCATCTAAAGCTAAATATTGGCGAGAGACCGATAGCGAACAAGTACCGTGAGGGAAAGATGAAAAGC ACTTTGGAAAGAGAGTTAAACAGTACGTGAAATTGCTGAAAGGGAAACGCTTGAAGTCAGTCGCGTCT GCCGGAACTCAGCCTTGCTTTTGCTTGGTGCACTTTCCGGTTGACGGGCCAGCATCGATTTTGACCGTC GGAAAAGGGCTGGAGAAATGTGGCACCTCCGGGTGTGTTATAGTCTTCAGTCGTATACGGCGGTTGGG ATCGAGGAACGCAGCGCGCCGCAAGGCAGGGGTTCGCCCACTATCGCGCTTAGGATGCTGGCGTAAT GGCTTTAAACGACCCGTCTTG

PUB-2B_DR_S014381_B05_045 (602 bp)

GCGCGATAGTGGGCGAACCCCTGCCTTGCGGCGCGCTGCGTTCCTCGATCCCAACCGCCGTATACGAC TGAAGACTATAACACACCCGGAGGTGCCACATTTCTCCAGCCCTTTTCCGACGGTCAAAATCGATGCT GGCCCGTCAACCGGAAAGTGCACCAAGCAAAAGCAAGGCTGAGTTCCGGCAGACGCGACTGACTTCA AGCGTTTCCCTTTCAGCAATTTCACGTACTGTTTAACTCTCTTTCCAAAGTGCTTTTCATCTTTCCCTCA CGGTACTTGTTCGCTATCGGTCTCTCGCCAATATTTAGCTTTAGATGGAATTTACCACCCATTTTGAGCT GCATTCCCAAACAACTCGACTCTTTGAGAGCGCATCACAAAGCACTGGTAGTCCGTGTCAAAGACGGG ATTCTCACCCTCTACGACGCTCTGTTCCAAGAGACTTATACACGGTCCAGCGCGGAAAGCACTTCTCCA GACTACAACTCGGACGGCCAAAGACCGCCAGATTTTAAATTTGAGCTTTTCCCGCTTCACTCGCAGTTA CTAGGGGAATCCTTGTTAGTTTCTTTTCCTCCGCTTATTGATATGCTTAATAA

\section{Consensus Sequence PUB (641 bp)}

TTATTAAGCATATCAATAAGCGGAGGAAAAGAAACTAACAAGGATTCCCCTAGTAACTGCGAGTGAAGC GGGAAAAGCTCAAATTTAAAATCTGGCGGTCTTTGGCCGTCCGAGTTGTAGTCTGGAGAAGTGCTTTCC GCGCTGGACCGTGTATAAGTCTCTTGGAACAGAGCGTCGTAGAGGGTGAGAATCCCGTCTTTGACACGG ACTACCAGTGCTTTGTGATGCGCTCTCAAAGAGTCGAGTTGTTTGGGAATGCAGCTCAAAATGGGTGGT AAATTCCATCTAAAGCTAAATATTGGCGAGAGACCGATAGCGAACAAGTACCGTGAGGGAAAGATGAA AAGCACTTTGGAAAGAGAGTTAAACAGTACGTGAAATTGCTGAAAGGGAAACGCTTGAAGTCAGTCGC GTCTGCCGGAACTCAGCCTTGCTTTTGCTTGGTGCACTTTCCGGTTGACGGGCCAGCATCGATTTTGACC GTCGGAAAAGGGCTGGAGAAATGTGGCACCTCCGGGTGTGTTATAGTCTTCAGTCGTATACGGCGGTTG GGATCGAGGAACGCAGCGCGCCGCAAGGCAGGGGTTCGCCCACTATCGCGCTTAGGATGCTGGCGTAA TGGCTTTAAACGACCCGTCTTG

Sequence data of the sample PUD

1. PUD-2H_DF_S014381_G04_020.ab1: Data obtained with Forward primer 2. PUD-2H_DR_S014381_H04_018.ab1: Data obtained with Reverse primer PUD-2H_DF_S014381_G04_020(520 bp)

GCTGGCTCCTTCGGAGTCCGCATTGTAATTTGCAGAGGATGCTTTGGGTGCGGCCCCCGTCTAAGTGCC CTGGAACGGGCCGTCAGAGAGGGTGAGAATCCCGTCTTGGGCGGGGTGTCCGTGCCCGTGTAAAGCTC CTTCGACGAGTCGAGTTGTTTGGGAATGCAGCTCTAAATGGGTGGTAAATTTCATCTAAAGCTAAATA CTGGCCGGAGACCGATAGCGCACAAGTAGAGTGATCGAAAGATGAAAAGCACTTTGAAAAGAGAGTT AAACAGCACGTGAAATTGTTGAAAGGGAAGCGCTTGCGACCAGACTCGCCCGCGGGGTTCAGCCGGC ATTCGTGCCGGTGTACTTCCCCGTGGGCGGGCCAGCGTCGGTTTGGGCGGCCGGTCAAAGGCCCCTGG AATGTAGTGCCCTCCGGGGCACCTTATAGCCAGGGGTGCAATGCGGCCAGCCTGGACCGAGGAACGC GCTTCGGCACGGACGCTGGCATAATGGTCGTAAACGACCCGTCTTG 


\section{PUD-2H_DR_S014381_H04_018(544 bp)}

GGCTGGCCGCATTGCACCCCTGGCTATAAGGTGCCCCGGAGGGCACTACATTCCAGGGGCCTTTGACCG GCCGCCCAAACCGACGCTGGCCCGCCCACGGGGAAGTACACCGGCACGAATGCCGGCTGAACCCCGC GGGCGAGTCTGGTCGCAAGCGCTTCCCTTTCAACAATTTCACGTGCTGTTTAACTCTCTTTTCAAAGTGC TTTTCATCTTTCGATCACTCTACTTGTGCGCTATCGGTCTCCGGCCAGTATTTAGCTTTAGATGAAATTTAC CACCCATTTAGAGCTGCATTCCCAAACAACTCGACTCGTCGAAGGAGCTTTACACGGGCACGGACACCC CGCCCAAGACGGGATTCTCACCCTCTCTGACGGCCCGTTCCAGGGCACTTAGACGGGGGCCGCACCCA AAGCATCCTCTGCAAATTACAATGCGGACTCCGAAGGAGCCAGCTTTCAAATTTGAGCTCTTGCCGCTT CACTCGCCGTTACTGAGGCAATCCCGGTTGGTTTCTTTTCCTCCGCTTATTGATATGCTT

\section{Consensus Sequence PUD (632 bp)}

TTTGGGTCCGTTGTTCAAGACGGGCGGATTACGACCATTACGCCAGCATCCTAGCCGAAGCGCGGACCT CGCCCGGCGCTCGCTGCATCACAAGAGGCTATAACACTCCCGAGGGAGCTACATTCCTCAAGTCTTATC CAGCGGCACCAGACGATGCTGGCCTGCAACGCGGCGAGTAGACCGGTCAGAAGACCGGCGGAACACC GCGAGCAAGTCTGGTTGCAATCCCTTCCCTTTTAACAATTTCACGTGCTTTTTAACTCTCTTTCCAAAGT GCTTTTCATCTTTCGATCACTCTACTTGTGCGCTATCGGTCTCTGGCCAATATTTAGCTTTAGAAGAAATTT ACCTCCCATTTAGAGCTGCATTCCCAAACAACTCGACTCGTCGAAGGAGCTACGTATAGAGCACCTTTC CGACCGCATACGGGATTCTCACCCTCTATGACGTCCTGTTCCAAGGAACTTAGGTCGGTGGTTACTCAGA AGCATCCTCTACAAATTACAACTCGGACGTCGAAGACGCCAGATTTCAAATTTGAGCTATTGCTGCTTCA CTCGCCGTTACTAGAGCAATCCCTGTTGGTTTCTTTTCCTCCGCTTATTGATATGCTTAAGTCCCACCGGG GTAAA

\section{Distribution of 101 Blast Hits on the Query Sequence}

\begin{tabular}{|c|c|c|c|c|c|c|}
\hline ect 센 Hone Selected:-15 & & & & & & \\
\hline 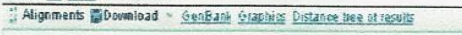 & & & & & & \\
\hline Dess & $\begin{array}{l}\text { Max } \\
\text { scone }\end{array}$ & $\begin{array}{l}\text { Total } \\
\text { scove }\end{array}$ & $\begin{array}{l}\text { Ouery } \\
\text { conet. }\end{array}$ & $\underset{\text { ralue }}{E}$ & ient & \\
\hline 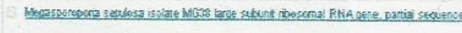 & 1147 & 1728 & $96 \%$ & 0.0 & $\cos$ & Eusbeachi: \\
\hline 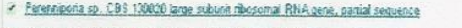 & 1142 & 1142 & $\infty x$ & 0.0 & $100 \%=$ & 10001 \\
\hline 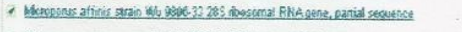 & 1123 & 1123 & $\infty 6$ & 0.0 &  & Avasos311 \\
\hline 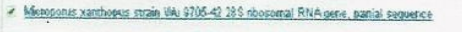 & 1118 & 1118 & 996 & 0.0 & $\infty \times$ & Arsango.1 \\
\hline  & 1112 & 1112 & 208 & 0.0 & $20 \%$ & Arsoles: 1 \\
\hline 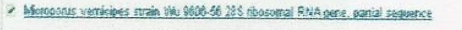 & 1112 & 1112 & 998 & 00 & $\infty x=$ & Arstsal \\
\hline  & 1107 & 1107 & $98 \%$ & 0.0 & $98 \%$ & Anseses: \\
\hline 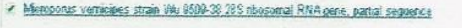 & 1103 & 1103 & $\infty \%$ & 0.0 & $\operatorname{sex}=$ & AV318001 \\
\hline 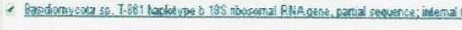 & 1101 & 1101 & $\infty 08 \%$ & 0.0 & $\infty \times 1$ & ke \\
\hline 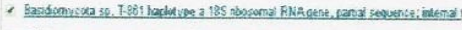 & 1101 & 1101 & 905 & 0.0 & $\infty \times 1$ &  \\
\hline  & 1101 & 1101 & $\cos$ & 0.0 & $90 \times 5$ & Ho65620241 \\
\hline  & 1101 & 1101 & 905 & 0.0 & $98 \%$ E & Everses: \\
\hline  & 1101 & 1101 & 998 & 0.0 & $98 \times 6$ & Eves:580. \\
\hline 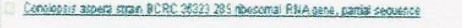 & 1801 & 1101 & $\infty \%$ & 0.0 & $985=$ & anstose 1 \\
\hline 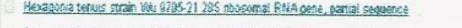 & 1101 & 1101 & $96 \%$ & 0.0 & $98 \% \leq$ &  \\
\hline  & 11011 & 1101 & $\operatorname{ses}$ & 0.0 & $98 \times 5$ & Donotas: \\
\hline 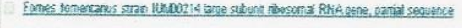 & 15011 & 1101 & $99 \%$ & 0.0 & 9965 & conereq4! \\
\hline 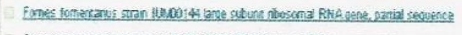 & 11011 & 1101 & 008 & 0.0 & $20 \times 5$ & consestis 1 \\
\hline  & 10281 & 1009 & $97 \times$ & 0.0 & $98 \times \leq$ & Ansogos: \\
\hline 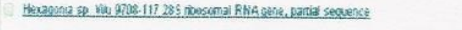 & 10991 & 1009 & 206 & 0.0 & $98 \%=$ & Ars61939. \\
\hline  & 10981 & 1098 & 298 & 0.0 & 985 & stupea : \\
\hline 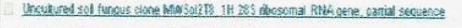 & 1090 & 1008 & $99 \%$ & 0.0 & $28 \times E$ & Eusosotis. \\
\hline 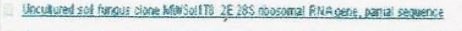 & 1090 & 1008 & $96 \%$ & 0.0 & 886 E & Eeser2519. \\
\hline 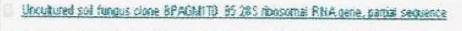 & 1000 & 1098 & 208 & 0.0 & 2856 & 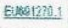 \\
\hline 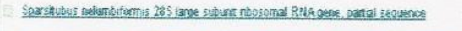 & 1090 & 1000 & $\cos x$ & 0.0 & 98*0 & 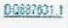 \\
\hline 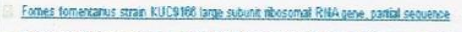 & 1056 & 1055 & 948 & 0.0 & $08 \times 1$ & .546079 .1$. \\
\hline 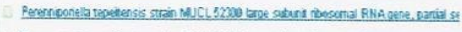 & 1055 & 1055 & 906 & 0.0 & $978 \mathrm{~b}$ & mearsis \\
\hline 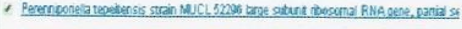 & 10561 & 1065 & 2006 & 0.0 & 978 & 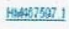 \\
\hline 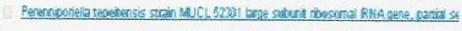 & 10561 & 1055 & $\operatorname{sex}$ & 0.0 & $97 \%$ ह & Heanse日 1 \\
\hline 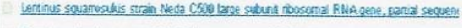 & 10561 & 1065 & $\infty$ & 0.0 & $98 \%$ s & A52603:1 \\
\hline 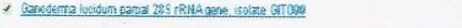 & 10531 & 1063 & $96 \%$ & 0.0 & 978 a & Ancos30.1 \\
\hline 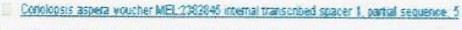 & 10511 & 1051 & 95x & 0.0 & $98 \% \mathrm{~K}$ & 19001727201 \\
\hline 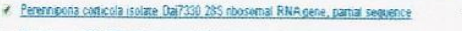 & 10511 & 1051 & 248 & 0.0 & $98 \% \mathrm{H}$ & H00591981 1 \\
\hline 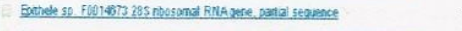 & 1051 & 1051 & $20 \%$ & 0.0 & $97 \%$ 므 & 000799 \\
\hline  & 1051 & 051 & 996 & 0.0 & $96 \times \mathrm{A}$ & Arassain: \\
\hline  & 1050 & 1050 & 36 & 0.0 & $28 \% \leq$ & jovgener \\
\hline
\end{tabular}

\begin{tabular}{|c|c|}
\hline \multicolumn{2}{|l|}{ 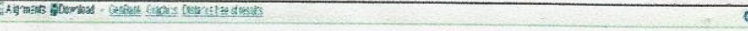 } \\
\hline Exte & 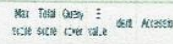 \\
\hline \multirow{2}{*}{\multicolumn{2}{|c|}{  }} \\
\hline & \\
\hline \multicolumn{2}{|c|}{ 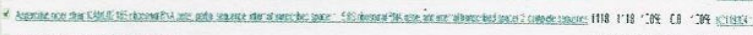 } \\
\hline  &  \\
\hline 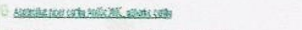 & 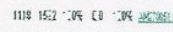 \\
\hline 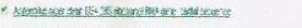 & 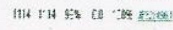 \\
\hline  & 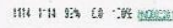 \\
\hline 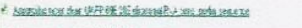 &  \\
\hline 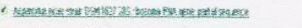 & 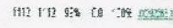 \\
\hline 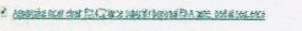 & 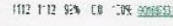 \\
\hline 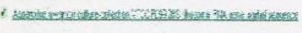 & 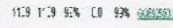 \\
\hline 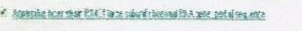 & 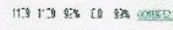 \\
\hline  & $15: 17.76090$ \\
\hline 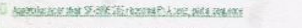 & 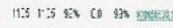 \\
\hline 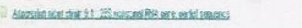 & 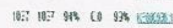 \\
\hline \multicolumn{2}{|c|}{ 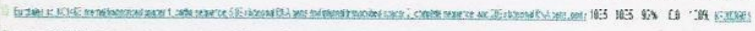 } \\
\hline \multicolumn{2}{|c|}{ 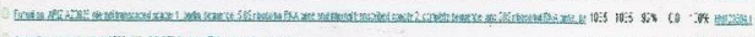 } \\
\hline  &  \\
\hline \multicolumn{2}{|c|}{ 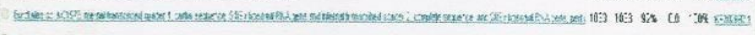 } \\
\hline \multicolumn{2}{|c|}{  } \\
\hline 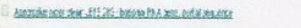 & 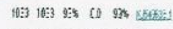 \\
\hline 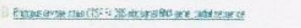 & 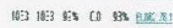 \\
\hline \multicolumn{2}{|c|}{  } \\
\hline  & 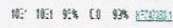 \\
\hline \multirow{2}{*}{\multicolumn{2}{|c|}{  }} \\
\hline & \\
\hline \multicolumn{2}{|c|}{  } \\
\hline 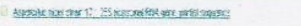 &  \\
\hline  & 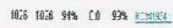 \\
\hline  & 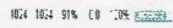 \\
\hline \multicolumn{2}{|c|}{ 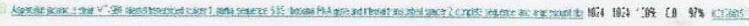 } \\
\hline 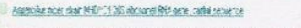 & 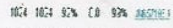 \\
\hline \multicolumn{2}{|c|}{  } \\
\hline 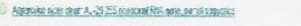 & is ongs: \\
\hline 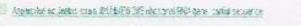 & 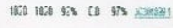 \\
\hline  &  \\
\hline 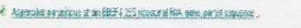 & 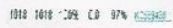 \\
\hline 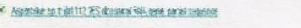 & 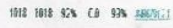 \\
\hline  &  \\
\hline 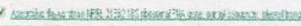 & \\
\hline
\end{tabular}


a

$\mathrm{b}$

BLAST DATA of PUB and PUD (Alignment view using combination of NCBI GenBank)

Significant Alignments Produced by the Sequence:

1. PUB

\begin{tabular}{|c|c|c|c|c|c|c|}
\hline Accession & Description & $\frac{\text { Max }}{\text { score }}$ & $\frac{\text { Total }}{\text { score }}$ & $\begin{array}{c}\text { Query } \\
\text { coverage }\end{array}$ & $\frac{\mathrm{E}}{\text { value }}$ & $\underline{\text { Max ident }}$ \\
\hline JX292098.1 & Perenniporia sp. CBS 130020 & 1142 & 1142 & $96 \%$ & 0.0 & $100 \%$ \\
\hline AY351931.1 & \begin{tabular}{|c|} 
Microporus affinis strain Wu 9806- \\
33
\end{tabular} & 1123 & 1123 & $99 \%$ & 0.0 & $98 \%$ \\
\hline AY333799.1 & $\begin{array}{l}\text { Microporus xanthopus strain } \mathrm{Wu} \\
9705-42\end{array}$ & 1118 & 1118 & $99 \%$ & 0.0 & $98 \%$ \\
\hline AY351942.1 & $\begin{array}{l}\text { Hexagonia hydnoides strain CRM- } \\
22\end{array}$ & 1112 & 1112 & $99 \%$ & 0.0 & $98 \%$ \\
\hline Y351929.1 & $\begin{array}{l}\text { Microporus vernicipes strain } \mathrm{Wu} \\
9606-56\end{array}$ & 1112 & 1112 & $99 \%$ & 0.0 & $98 \%$ \\
\hline AY351944.1 & Hexagonia hirta strain CBS 515.96 & 1107 & 1107 & $99 \%$ & 0.0 & $98 \%$ \\
\hline AY351930.1 & $\begin{array}{l}\text { Microporus vernicipes strain } \mathrm{Wu} \\
\text { 9509-38 }\end{array}$ & 1103 & 1103 & $98 \%$ & 0.0 & $98 \%$ \\
\hline KC176301.1 & $\begin{array}{l}\text { Basidiomycota } \mathrm{sp} \text {. T-861 haplotype } \\
\mathrm{b}\end{array}$ & 1101 & 1101 & $99 \%$ & 0.0 & $98 \%$ \\
\hline KC176300.1 & Basidiomycota sp. T-861 haplotype a & 1101 & 1101 & $99 \%$ & 0.0 & $98 \%$ \\
\hline HQ659224.1 & Dichomitus squalens voucher & 1101 & 1101 & $99 \%$ & 0.0 & $98 \%$ \\
\hline HM467597.1 & $\begin{array}{l}\text { Perenniporiella tepeitensis } \text { strain } \\
\text { MUCL } 52296\end{array}$ & 1055 & 1055 & $99 \%$ & 0.0 & $97 \%$ \\
\hline AM269830.1 & Ganoderma lucidum isolate GITO99 & 1053 & 1053 & $96 \%$ & 0.0 & $97 \%$ \\
\hline HQ654108.1 & $\begin{array}{c}\text { Perenniporia corticola isolate } \\
\text { Dai7330 }\end{array}$ & 1051 & 1051 & $94 \%$ & 0.0 & $98 \%$ \\
\hline AY333817.1 & $\begin{array}{c}\text { Coriolopsis polyzona strain OH-184- } \\
\text { SP }\end{array}$ & 1051 & 1051 & $99 \%$ & 0.0 & $96 \%$ \\
\hline JQ001847.1 & $\begin{array}{l}\text { Perenniporia aridula voucher Dai } \\
12398\end{array}$ & 1050 & 1050 & $93 \%$ & 0.0 & $98 \%$ \\
\hline
\end{tabular}

\section{PUD}

\begin{tabular}{|c|c|c|c|c|c|c|}
\hline Accession & Description & $\underline{\text { Max }}$ & $\begin{array}{l}\text { Total } \\
\underline{\text { score }}\end{array}$ & $\begin{array}{c}\text { Query } \\
\text { coverage }\end{array}$ & $\begin{array}{c}\underline{\mathrm{E}} \\
\text { value }\end{array}$ & Max ident \\
\hline KJ365316.1 & Aspergillus niger strain NJA-1 & 1118 & 1118 & $100 \%$ & 0.0 & $100 \%$ \\
\hline KF304798.1 & Aspergillus niger & 1118 & 1118 & $100 \%$ & 0.0 & $100 \%$ \\
\hline KC119204.1 & Aspergillus niger strain KAML02 & 1118 & 1118 & $100 \%$ & 0.0 & $100 \%$ \\
\hline JF521496.1 & Aspergillus niger strain US4 & 1114 & 1114 & $99 \%$ & 0.0 & $100 \%$ \\
\hline HM008328.1 & Aspergillus niger isolate BK 01 & 1114 & 1114 & $99 \%$ & 0.0 & $100 \%$ \\
\hline AY216672.1 & Aspergillus niger strain UWFP 696 & 1114 & 1114 & $99 \%$ & 0.0 & $100 \%$ \\
\hline
\end{tabular}




\begin{tabular}{|c|c|c|c|c|c|c|}
\hline Accession & Description & $\underline{\underline{\text { Max }}}$ & $\begin{array}{l}\text { Total } \\
\underline{\text { score }} \\
\end{array}$ & $\begin{array}{c}\text { Query } \\
\text { coverage }\end{array}$ & $\underline{\underline{\mathbf{E}}} \underline{\text { value }}$ & Max ident \\
\hline JX292268.1 & Aspergillus niger strain DSM 1627 & 1112 & 1112 & $99 \%$ & 0.0 & $100 \%$ \\
\hline GQ169753.1 & Aspergillus niger strain RA402 & 1112 & 1112 & $99 \%$ & 0.0 & $100 \%$ \\
\hline GQ892590.1 & $\begin{array}{l}\text { Aspergillus awamori culture-collection } \\
\text { VTCC:F099 }\end{array}$ & 1109 & 1109 & $99 \%$ & 0.0 & $99 \%$ \\
\hline GQ169752.1 & Aspergillus niger strain RA401 & 1109 & 1109 & $99 \%$ & 0.0 & $99 \%$ \\
\hline EU381181.1 & Aspergillus aculeatus strain NRRL 360 & 1020 & 1020 & $99 \%$ & 0.0 & $97 \%$ \\
\hline KC589438. & Aspergillus parasiticus strain BBEF4 & 1018 & 1018 & $100 \%$ & 0.0 & $97 \%$ \\
\hline JN967012.1 & Aspergillus sp. trijbl1112 & 1018 & 1018 & $92 \%$ & 0.0 & $97 \%$ \\
\hline HQ395773.1 & Aspergillus flavus isolate V5F-13 & 1018 & 1018 & $100 \%$ & 0.0 & $97 \%$ \\
\hline HQ856223.1 & Aspergillus flavus strain NRRL 21882 & 1018 & 1018 & $100 \%$ & 0.0 & $97 \%$ \\
\hline
\end{tabular}

Distance Matrix of the sample PUB and PUD

\begin{tabular}{|c|c|c|c|c|c|c|c|c|c|c|c|c|}
\hline & & 1 & 2 & 3 & 4 & 5 & 6 & 7 & 8 & 9 & 10 & 11 \\
\hline PUB & 1 & & 0.0000 & 0.0053 & 0.0057 & 0.0058 & 0.0061 & 0.0061 & 0.0061 & 0.0063 & 0.0063 & 0.0061 \\
\hline JX292098.1 & 2 & 0.0000 & & 0.0053 & 0.0057 & 0.0058 & 0.0061 & 0.0061 & 0.0061 & 0.0063 & 0.0063 & 0.0061 \\
\hline AY351931.1 & 3 & 0.0170 & 0.0170 & & 0.0016 & 0.0048 & 0.0024 & 0.0042 & 0.0024 & 0.0047 & 0.0047 & 0.0052 \\
\hline AY333799.1 & 4 & 0.0188 & 0.0188 & 0.0017 & & 0.0051 & 0.0016 & 0.0046 & 0.0016 & 0.0050 & 0.0050 & 0.0056 \\
\hline AY351942.1 & 5 & 0.0205 & 0.0205 & 0.0136 & 0.0153 & & 0.0055 & 0.0061 & 0.0055 & 0.0065 & 0.0065 & 0.0056 \\
\hline Y351929.1 & 6 & 0.0205 & 0.0205 & 0.0034 & 0.0017 & 0.0170 & & 0.0050 & 0.0000 & 0.0053 & 0.0053 & 0.0058 \\
\hline AY351944.1 & 7 & 0.0222 & 0.0222 & 0.0118 & 0.0135 & 0.0222 & 0.0153 & & 0.0050 & 0.0053 & 0.0053 & 0.0054 \\
\hline AY351930.1 & 8 & 0.0205 & 0.0205 & 0.0034 & 0.0017 & 0.0170 & 0.0000 & 0.0153 & & 0.0053 & 0.0053 & 0.0058 \\
\hline KC176301.1 & 9 & 0.0239 & 0.0239 & 0.0135 & 0.0153 & 0.0240 & 0.0170 & 0.0187 & 0.0170 & & 0.0000 & 0.0048 \\
\hline KC176300.1 & 10 & 0.0239 & 0.0239 & 0.0135 & 0.0153 & 0.0240 & 0.0170 & 0.0187 & 0.0170 & 0.0000 & & 0.0048 \\
\hline HQ659224.1 & 11 & 0.0239 & 0.0239 & 0.0170 & 0.0187 & 0.0204 & 0.0204 & 0.0187 & 0.0204 & 0.0136 & 0.0136 & \\
\hline
\end{tabular}

\begin{tabular}{|l|c|c|c|c|c|c|c|c|c|c|c|c|}
\hline & & 1 & 2 & 3 & 4 & 5 & 6 & 7 & 8 & 9 & 10 & 11 \\
\hline PUD & 1 & 0.0000 & 0.0000 & 0.0000 & 0.0000 & 0.0000 & 0.0000 & 0.0000 & 0.0000 & 0.0017 & 0.0000 & 0.0000 \\
\hline KJ365316.1 & 2 & 0.0000 & & 0.0000 & 0.0000 & 0.0000 & 0.0000 & 0.0000 & 0.0000 & 0.0000 & 0.0017 & 0.0000 \\
\hline KF304798.1 & 3 & 0.0000 & 0.0000 & & 0.0000 & 0.0000 & 0.0000 & 0.0000 & 0.0000 & 0.0000 & 0.0017 & 0.0000 \\
\hline KC119204.1 & 4 & 0.0000 & 0.0000 & 0.0000 & & 0.0000 & 0.0000 & 0.0000 & 0.0000 & 0.0000 & 0.0017 & 0.0000 \\
\hline JF521496.1 & 5 & 0.0000 & 0.0000 & 0.0000 & 0.0000 & & 0.0000 & 0.0000 & 0.0000 & 0.0000 & 0.0017 & 0.0000 \\
\hline HM008328.1 & 6 & 0.0000 & 0.0000 & 0.0000 & 0.0000 & 0.0000 & & 0.0000 & 0.0000 & 0.0000 & 0.0017 & 0.0000 \\
\hline AY216672.1 & 7 & 0.0000 & 0.0000 & 0.0000 & 0.0000 & 0.0000 & 0.0000 & & 0.0000 & 0.0000 & 0.0017 & 0.0000 \\
\hline JX292268.1 & 8 & 0.0000 & 0.0000 & 0.0000 & 0.0000 & 0.0000 & 0.0000 & 0.0000 & & 0.0000 & 0.0017 & 0.0000 \\
\hline GQ169753.1 & 9 & 0.0000 & 0.0000 & 0.0000 & 0.0000 & 0.0000 & 0.0000 & 0.0000 & 0.0000 & & 0.0017 & 0.0000 \\
\hline GQ892590.1 & 10 & 0.0018 & 0.0018 & 0.0018 & 0.0018 & 0.0018 & 0.0018 & 0.0018 & 0.0018 & 0.0018 & & 0.0017 \\
\hline GQ169752.1 & 11 & 0.0000 & 0.0000 & 0.0000 & 0.0000 & 0.0000 & 0.0000 & 0.0000 & 0.0000 & 0.0000 & 0.0018 & \\
\hline
\end{tabular}




\section{Phylogenetic Tree:}

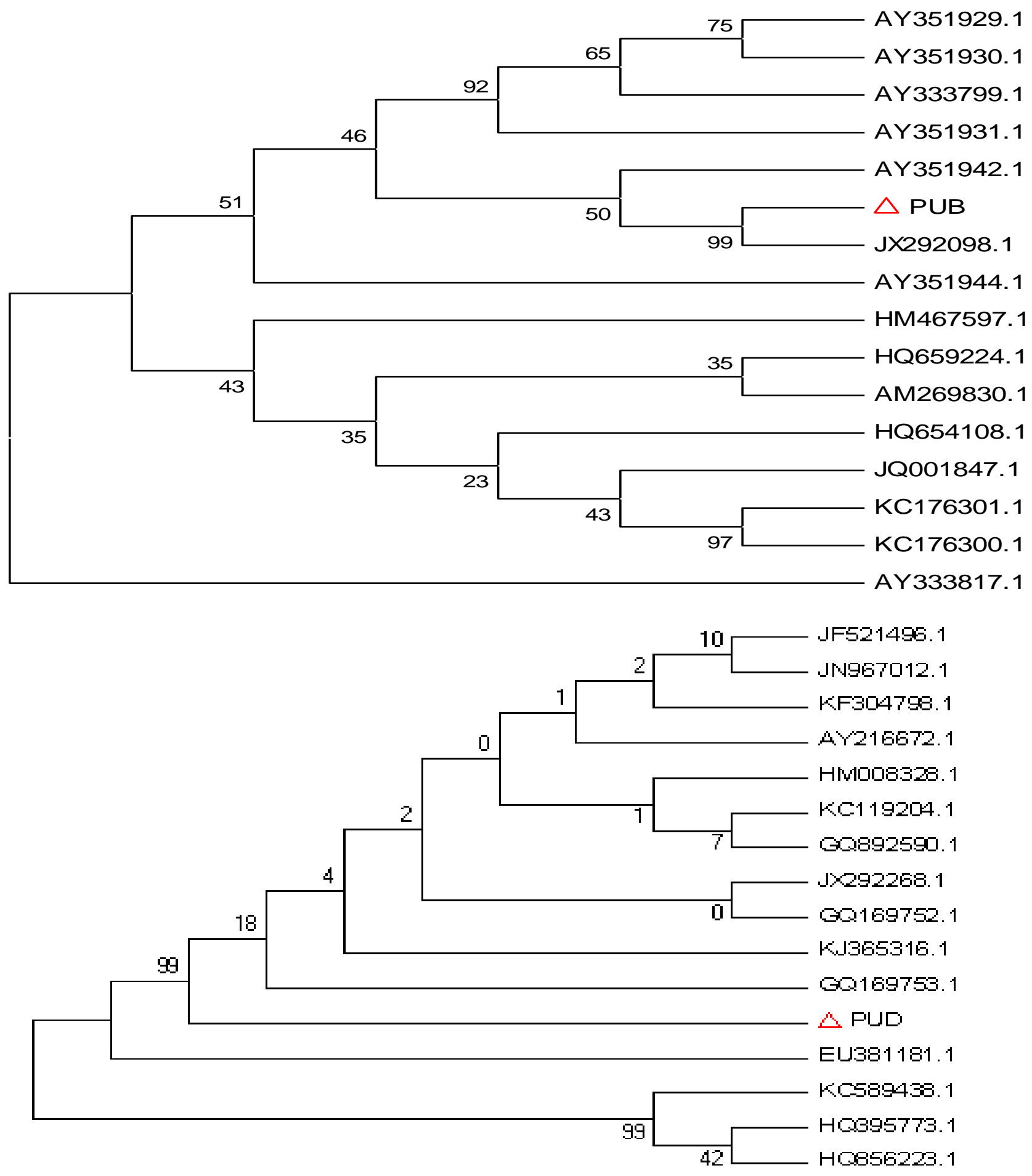






PUB

PUD

Microscopic view of the samples

Identification of fungi using conventional methods like growth characteristics, morphology, colony pattern, and biochemical reactions may be difficult. DNA sequencing of ribosomal RNA genes is becoming the most appropriate alternative to the conventional methods for identifying fungi. The ribosomal RNA genes exhibit markedly variable sequences when comparing between different species. These genes also have highly conserved sequences between all fungi, and these regions are used as primer binding sites for amplification and sequencing reactions. Fungal genomic DNA is extracted from a pure culture and amplified using PCR reaction. Sequencing results are used to search databases of verified fungal sequences and the percent match to the top hits is calculated and evaluated along with phylogenetic trees of relatedness. These results are compared to the traditional methods used in identification of the organisms. In present investigation, the culture, labeled as PUB exhibited similarity to Perenniporia sp. CBS 130020 GenBank Accession Number: JX292098.1) and the culture, PUD to Aspergillus niger strain RA402 (GenBank Accession Number: GQ169753.1) based on nucleotide homology and phylogenetic analysis.

\section{Acknowledgement}

The financial assistance received from The UGC, New Delhi in the form of CAS (Phsae- II) under SAP programme is thankfully acknowledged by the authors.

\section{References}

Felsenstein, J. 1985. Confidence limits on phylogenies: An approach using the bootstrap. Evol., 39: 783-791.

Harley, J.L., Smith, S.E. 1983. Mycorrhizal symbiosis. Academic Press, New York.

Haselwandter, K., Read, D.J. 1982. The significance of root-fungus association in two Carex species of high-alpine plant communities. Oecologia, 53: 352-354.

Jumpponen, A. 2001. Dark septate endophytes - are they mycorrhizal? Mycorrhiza, 11: 207-211.

Jumpponen, A., Trappe, J.M. 1998. Dark septate endophytes: a review of facultative biotrophic root colonizing fungi. New Phytol., 140: 295-310.

Kimura, M. 1980. A simple method for estimating evolutionary rate of base substitutions through comparative 
studies of nucleotide sequences. $J$. Mol. Evol., 16: 111-120.

Newsham, K.K. 1999. Phialophora graminicola, a dark septate fungus, is a beneficial associate of the grass Vulpia ciliata ssp. ambiqua. New Phytol., 144: 517-524.

Saitou, N. and Nei, M. 1987. The neighborjoining method: A new method for reconstructing phylogenetic trees. Mol. Biol. Evol., 4: 406-425.

Smith, S.E., Read, D.J. 1997. Mycorrhizal symbiosis. 2nd edn. Academic Press, London.
Tamura, K., Peterson, D., Peterson, N., Stecher, G., Nei, M., Kumar, S. 2011. MEGA5: Molecular Evolutionary Genetics Analysis using Maximum Likelihood, Evolutionary Distance, and Maximum Parsimony Methods. Mol. Biol. Evol., 28: 2731-27.

Yu, T., Nassuth, A., Peterson, R.L. 2001. Characterization of the interaction between the dark septate fungus Phialocephala fortinii and Asparagus officinalis roots. Can. J. Bot., 47: 741753.

\section{How to cite this article:}

Avishek Sarkar and Sikha Dutta. 2016. Isolation and Identification of Some Dark Septate Endophytic Fungi (DSE) from the Roots of the Ethno-Medicinal Plant Plumbago zeylanica L. Int.J.Curr.Microbiol.App.Sci. 5(10): 698-707. doi: http://dx.doi.org/10.20546/ijcmas.2016.510.076 